\title{
Pacific
}

Journal of

Mathematics

\section{DEHN FILLING HYPERBOLIC 3-MANIFOLDS}

COLin C. AdAms 


\title{
DEHN FILLING HYPERBOLIC 3-MANIFOLDS
}

\author{
Colin AdAMS
}

\begin{abstract}
Define a complete family of parent (ancestor) manifolds to be a set of compact 3-manifolds such that every closed orientable 3-manifold can be obtained by one (or more) Dehn fillings of the manifolds in the family. In 1983, R. Myers proved that the set of 1-cusped hyperbolic 3manifolds is a complete family of parent manifolds. We prove this result in a new way and then go on to prove:
\end{abstract}

THEOREM 1.1. (a) Let $V_{0}$ be any positive real number. Then the set of 1-cusped hyperbolic 3-manifolds of volume greater than $V_{0}$ is a complete family of parent manifolds.

(b) Let $V_{1}$ be any positive real number. Then the set of 1-cusped hyperbolic 3-manifolds of cusp volume greater than $V_{1}$ is a complete family of parent manifolds.

(c) The set of 2-cusped hyperbolic 3-manifolds containing embedded totally geodesic surfaces is a complete family of ancestor (actually grandparent) manifolds.

(d) For any positive integer $N$, the set of hyperbolic 3-manifolds, each of which shares its volume with $N$ or more other hyperbolic 3-manifolds, is a complete family of ancestor manifolds.

As a corollary to Theorem 1(b), we prove that there exists a complete family of parent manifolds such that at most one Dehn filling on each manifold in the family yields a manifold of finite fundamental group.

1. Introduction. A Dehn filling of a 3-manifold with a torus boundary component is the procedure of gluing a solid torus to the 3 -manifold along the torus boundary. If a manifold $M$ is obtained from a manifold $M^{\prime}$ by a single Dehn filling, we will call $M^{\prime}$ a parent of $M$. If $M$ is obtained from $M^{\prime}$ by some number of Dehn fillings, we will call $M^{\prime}$ an ancestor of $M$. We will define a set of 3 -manifolds to 
be a complete family of parent (ancestor) manifolds if every closed orientable 3-manifold has a parent (ancestor) in the set.

In [14] and [21], Lickorish and Wallace independently proved that the set of link exteriors in the 3 -sphere is a complete family of ancestor manifolds. (A link exterior is the complement of an open regular neighborhood of the link.) It follows that if $M$ is any compact orientable 3-manifold without boundary, the set of all link exteriors in $M$ is a complete family of ancestor manifolds.

In [20] (see also [2]) the set of hyperbolic link exteriors in $S^{3}$ was shown to be a complete family of ancestor manifolds. Alexander [3] proved that compact orientable surface bundles over $S^{1}$ with torus boundary components form a complete family of ancestor manifolds. Myers [15] and Gonzalez-Acuna [7] showed that in fact compact orientable surface bundles over $S^{1}$ with a single torus boundary component form a complete family of parent manifolds.

In 1983, Myers [16] proved that the set of 1-cusped orientable hyperbolic 3-manifolds is a complete family of parent manifolds. Note that this is equivalent to showing that any closed orientable manifold contains a knot with hyperbolic complement. (Recently, Myers has extended his result to also hold for closed nonorientable 3-manifolds, see [17]). Myer's proof is similar in spirit to the original proof of Bing's Theorem. Bing's Theorem states that if $M$ is a closed orientable 3 -manifold and every simple closed curve in $M$ is contained in a 3 -ball in $M$, then $M$ is homeomorphic to the 3 -sphere (cf. [4] or [18]). In both proofs, the dual 1-skeleton of a triangulation of the manifold is approximated by a simple closed curve, where the vertices are replaced by appropriately chosen tangles.

In this paper, we will reprove Myer's result from a completely different point of view. This point of view will also allow us to prove the following:

\section{THEOREM 1.1.}

(a) Let $V_{0}$ be any positive real number. Then the set of 1-cusped hyperbolic 3-manifolds of volume greater than $V_{0}$ is a complete family of parent manifolds.

(b) The $V_{1}$ be any positive real number. Then the set of 1-cusped hyperbolic 3-manifolds of cusp volume greater than $V_{1}$ is a complete family of parent manifolds. 
(c) The set of 2-cusped hyperbolic 3-manifolds containing embedded totally geodesic surfaces is a complete family of ancestor (actually grandparent) manifolds.

(d) For any positive integer $N$, the set of hyperbolic 3-manifolds, each of which shares its volume with $N$ or more other hyperbolic 3-manifolds, is a complete family of ancestor manifolds.

In order to prove these results, we will first prove that for any dis= joint pair of nontrivial boundary curves $\phi_{1}$ and $\phi_{2}$ in a compact orientable 3-manifold $M$ with finite volume hyperbolic interior, there exists a knot $g$ in $M$ such that $M-\stackrel{\circ}{N}(g)$ has hyperbolic interior and contains an essential pair of pants with $\phi_{1}$ and $\phi_{2}$ as two boundary components. This will be the contents of $\S 2$. The results listed above will then follow from results in [1] and [2].

In addition to proving these results in $\S 3$, we include several corollaries. In particular, we obtain a topological corollary to Theorem 1.1b). There is a complete family of parent manifolds such that at most one Dehn filling on each manifold yields a closed manifold of finite fundamental group. We also see that any closed 3-manifold is obtained from Dehn filling a one-cusped hyperbolic 3-manifold such that the Dehn filling is along the shortest curve in a cusp boundary.

All hyperbolic 3-manifolds under consideration will be orientable finite volume noncompact complete hyperbolic 3 -manifolds. This implies that each such is the interior of a compact 3-manifold with nonempty boundary, all components of which are tori. (See [19]). The cusp volume of a hyperbolic 3-manifold with a single cusp is the largest possible volume that can be put in an embedded cusp. It corresponds to the volume of the cusp obtained as the projection of a set of horoballs in $H^{3}$, with disjoint interiors but with points of tangency.

A Dehn surgery with surgery coefficients $(p, q)$ along a link component $L$ will mean the removal of a regular neighborhood of the link and then a Dehn filling of the result along the torus boundary introduced, such that a meridian of the solid torus is glued to a simple closed curve in the homotopy class $[p m+q l]$ where $m$ is a meridian and $l$ is a longitude.

Definitions of incompressible, boundary incompressible, irreducible, boundary irreducible and Haken 3-manifold can be found in 
[10] and [13]. Thanks to Joel Hass, Alan Reid and the referee for conversations, comments and suggestions.

2. Essential Arcs. Let $M$ be a compact 3-manifold with nonempty boundary. A surface properly embedded in $M$ is said to be essential if it is incompressible and boundary-incompressible. An arc $\alpha$ that is properly embedded in $M$ is said to be essential in $M$ if it cannot be homotoped into $\partial M$. We will call a compact planar surface with three boundary components a pair of pants. If an arc $\alpha$ is properly embedded in a pair of pants $P$ and a is not isotopic in $P$ into $\partial P$, then we say that $\alpha$ is essential in $P$. We will utilize the following theorem.

THEOREM 2.1. [2; Theorem 3.1] Let $M$ be a compact orientable 3-manifold with non-empty boundary such that $\stackrel{\circ}{M}$ is a finite volume hyperbolic 3-manifold. Let $K$ be a knot in $\stackrel{\circ}{M}$ such that

(1) There does not exist $S^{1} \times S^{1} \times I \subseteq M$ such that $K \subseteq S^{1} \times S^{1} \times I$ where $S^{1} \times S^{1} \times\{0\} \subseteq \partial M$.

(2) There exists an essential pair of pants $P$ properly embedded in $M-\stackrel{\circ}{N}(K)$ such that exactly one component $\beta$ of $\partial P$ is contained in $\partial N(K)$.

(3) $K$ is not a torus knot in a solid torus $V \subseteq \stackrel{\circ}{M}$ such that $\partial V$ is incompressible in $M-\stackrel{\circ}{V}$.

(4) There does not exist an essential torus in $M^{\prime}=M-\stackrel{\circ}{N}(K)$ which bounds a knot exterior in $M^{\prime}$.

Then $M^{\prime}=M-\stackrel{\circ}{N}(K)$ has a hyperbolic interior.

We will use this theorem to prove the following.

THEOREM 2.2. Let $M$ be a compact 3-manifold with boundary such that $\stackrel{\circ}{M}$ is a finite volume hyperbolic 3-manifold. Let $\alpha$ be an arc that is properly embedded in $M$. Let $\phi_{1}$ and $\phi_{2}$ be non-intersecting nontrivial simple closed curves in $\partial M$ such that $\phi_{1}$ begins and ends at one endpoint of $\alpha$ and $\phi_{2}$ begins and ends at the other endpoint of $\alpha$. Suppose also that: 
(i) $\alpha$ is essential in $M$.

(ii) There does not exist a knot exterior in $M-\stackrel{\circ}{N}(\alpha)$ such that its boundary torus is incompressible.

Then there exists a simple closed curve $g$ in $M$ such that $M-$ $\stackrel{\circ}{N}(g)$ is a finite volume hyperbolic 3-manifold and $\alpha$ is essential in an essential pair of pants in $M-\stackrel{\circ}{N}(g)$ with two of its boundary components being $\phi_{1}$ and $\phi_{2}$.

Proof. We will begin by demonstrating that there is a choice of simple closed curve $\mu$ in $M$ that will allow the existence of an essential pair of pants $P$ in $M-\stackrel{\circ}{N}(\mu)$ such that $P$ contains $\alpha$ essentially and such that $\phi_{1}$ and $\phi_{2}$ are two of its boundary components. Note that in the case both of the endpoints of $\alpha$ begin and end on the same torus boundary component of $M, \phi_{1}$ and $\phi_{2}$ will be parallel curves on this torus.

Let $S$ be the boundary of a regular neighborhood of $\partial M \cup \alpha$. Define a simple closed curve $\mu$ in $S$ that is parallel to $\phi_{1}$ and $\phi_{2}$ when in $\partial(N(\partial M))$ and that is two parallel copies of $\alpha$ when in $\partial(N(\alpha))$. Note that by allowing the two strands of $\mu$ to twist around $\alpha$ as we move along $\partial(N(\alpha))$, we actually have an infinite family of possible choices for $\mu$.

Note also that for any choice of $\mu$, there is a pair of pants $P$ properly embedded in $M-\stackrel{\circ}{N}(\mu)$ with boundary components $\phi_{1}, \phi_{2}$ and a simple closed curve $\sigma$ in $\partial N(\mu)$. We can and do choose $P$ to contain $\alpha$. We will first prove that $P$ is an essential surface in $M-\stackrel{\circ}{N}(\mu)$.

If $P$ compresses, one of the three boundary components of $P$ must be trivial in $M$. Since $\partial M$ is incompressible, it must be that $\sigma$ is trivial in $M$. However, we can then glue a disk onto $\sigma$ in order to obtain from $P$ an annulus $A$ in $M$. Since the annulus is incompressible in $M$, it must be boundary compressible in $M$. Since $M$ is irreducible, $A$ must in fact be boundary-parallel in $M$. However, $A$ contains $\alpha$, contradicting the fact that $\alpha$ cannot be homotoped into $\partial M$. Thus, $P$ is incompressible.

Suppose now that $P$ is boundary compressible. This means that there exists an arc $j$ properly embedded in $P$ such that it does not cut a disk from $P$ and such that it forms half of the boundary of 
a disk $D$ in $M$, the other half being in $\partial M$. Since $\alpha$ cannot be homotoped into $\partial M, j$ cannot run from $\phi_{1}$ to $\phi_{2}$. Nor can $j$ begin and end on a single one of these boundary components as then we would be able to form an annulus in $M$ containing $\alpha$. The annulus would again be boundary-parallel, contradicting the fact $\alpha$ cannot be homotoped into the boundary.

The only remaining possibility is that $j$ begins and ends on $\sigma$, separating $\phi_{1}$ from $\phi_{2}$. Let $k$ be the complement of $\stackrel{\circ}{j}$ in $\partial D$. Since $k$ will only intersect one component of $N(P)-P, k$ intersects only one component of $N(\sigma)-\sigma$ on $\partial(N(\mu))$. Hence, $k$ can be isotoped into $\sigma$ in $\partial(N(\mu))$. However, we can then form a disk in $M$ with boundary one of $\phi_{1}$ or $\phi_{2}$, a contradiction to the fact $M$ must be boundary-irreducible.

Thus, $P$ is an essential pair of pants in $M-\stackrel{\circ}{N}(\mu)$ that contains $\alpha$ essentially. However, it is not necessarily the case that $M-\stackrel{\circ}{N}(\mu)$ has hyperbolic interior. We may have to exchange $\mu$ for a different curve in $M$. Theorem 2.1 will direct us toward an appropriate curve.

If we replace $K$ in Theorem 2.1 with our curve $\mu$, we have already shown that condition (b) of the hypotheses is satisfied. However, condition (a) may not be satisfied. We will show that by choosing a $\mu$ that twists appropriately about $\alpha$, we can make sure that condition (a) is satisfied.

Suppose that our first choice of $\mu$ does not satisfy condition (a), that is to say that $\mu$ lies in an $S^{1} \times S^{1} \times I$, where $S^{1} \times S^{1} \times\{0\}$ is a boundary component of $M$. Utilizing the hyperbolic structure on the interior of $M$, we realize the fundamental group of $M$ as a discrete group of isometries on hyperbolic 3-space $H^{3}$. Since $\mu$ is homotopic into $\partial M$, it lifts to a parabolic isometry of $H^{3}$. However, since each of $\phi_{1}$ and $\phi_{2}$ lifts to parabolic isometries of $H^{3}$, we have the parabolic isometry corresponding to $\mu$ that is the product of the two parabolic isometries corresponding to $\phi_{1}$ and $\phi_{2}$. In particular, this implies that $\phi_{1}$ and $\phi_{2}$ generate a Fuchsian subgroup of the fundamental group, that Fuchsian subgroup corresponding to a totally geodesic possibly immersed thrice-punctured sphere (see [1]).

However, since the isometry corresponding to $\phi_{1} \circ \phi_{2}^{-1}$ in the fundamental group of a thrice-punctured sphere is never parabolic, but rather is a non-rotating loxodromic isometry, any loop homo- 
topic to $\phi_{1} \circ \phi_{2}^{-1}$ cannot be contained in an $S^{1} \times S^{1} \times I$ such that $S^{1} \times S^{1} \times\{0\} \subseteq \partial M$. Taking any new choice for $\mu$ that twists an odd number of half-twists about $\alpha$ relative to the current $\mu$ will insure that this is the case.

It may be the case that $\mu$ does not satisfy condition (c) of Theorem 2.1. However, if $\mu$ is a torus knot in a solid torus $V$ such that $\partial V$ is incompressible in $M-\stackrel{\circ}{V}$, we will replace $\mu$ by the simple closed curve $g$ that is the core curve of the solid torus. We will take $N(g)$ to be the regular neighborhood of $g$ such that $\mu$ is a $(p, q)$-curve slightly inside $\partial V$, where $q>1$. We will examine the intersection curves in $P \cap \partial V$.

Suppose that there exists an intersection curve on $P$ concentric with $\phi_{i}$, where $i=1$ or 2 . Then $\phi_{i}$ is one boundary component of an annulus $A$ in $M$, such that the other boundary is a nontrivial $(r, s)$-curve $\psi$ on $\partial V$. The curve $\psi$ cannot be a meridian on $\partial V$ as if it were, $\phi_{i}$ would compress in $M$. If $R$ is the boundary component of $\partial M$ that contains $\phi_{i}$, then $V \cup N(A) \cup N(R)$ is a solid torus such that the regular neighborhood of an $(r, s)$-curve inside it has been removed. Let $Y$ be the outer boundary of this solid torus. If $s=1$, then $V \cup N(A) \cup N(R)$ is homeomorphic to $S^{1} \times S^{1} \times I$. Since it contains $\mu$, this would be a contradiction.

If $s \geq 2$, then $Y$ is both incompressible and not boundary-parallel to the $V \cup N(A) \cup N(R)$ side. If $Y$ compresses to the other side, it must bound a solid torus in $M$ to that side. Let $(u, v)$ be the compressing curve on $Y$. Assuming that $(r, s) \neq(u, v)$, we easily obtain a Seifert fibering of $M$ (or an essential annulus), a contradiction. If $(r, s)=(u, v)$, then $\phi_{i}$ compresses in $M$, again a contradiction.

If $Y$ is incompressible to the other side, it must be boundaryparallel to this side. Hence, $M$ is homeomorphic to $V \cup N(A) \cup N(R)$. However, $M$ would then again be Seifert fibered, a contradiction.

Thus, all intersection curves on $P$ must be concentric with $\sigma$. Since $\sigma$ lies in a distinct component of $M-\partial V$ from $\phi_{1}$ and $\phi_{2}$, there must be an odd number of intersection curves concentric with $\sigma$. We will replace $P$ by the pair of pants within $P$ bounded by $\phi_{1}$, $\phi_{2}$ and the intersection curve on $P$ furthest from $\sigma$. This new pair of pants, which we will continue to denote by $P$, will be properly embedded in $M-\stackrel{\circ}{V}$. If $g$ is the core curve of $V$, we think of $M-\stackrel{\circ}{V}$ 
as $M-\stackrel{\circ}{N}(g)$. It is not hard to check that $P$ remains essential in $M-\stackrel{\circ}{N}(g)$, and conditions (a) and (b) are still satisfied.

We also need to check that condition (c) is now satisfied, namely that $g$ itself cannot be a $(u, v)$-curve on a solid torus $W$ such that $\partial W$ is incompressible in $M-\stackrel{\circ}{W}$. If $g$ were a $(u, v)$-curve on a solid torus, then $\sigma$ would be a nontrivial cable on a torus knot. Hence, we could isotope $\sigma$ so that it was a nontrivial cable on a torus knot inside $W$. We can again prove that there cannot be an intersection curve concentric with $\phi_{1}$ or $\phi_{2}$ on $P$. Thus there must be an intersection curve concentric with $\sigma$ on $P$. But then $\sigma$ is isotopic to $\partial W$ in $W$, contradicting the fact it is a nontrivial cable on a torus knot.

Finally, we check that Condition (d) holds, namely that there does not exist an essential torus $T$ in $M^{\prime}=M-\stackrel{\circ}{N}(g)$ which bounds a knot exterior $Q$ in $M^{\prime}$. If this were the case, then $T$ would either be compressible or boundary-parallel in $M$. By condition (a) above, $T$ cannot be boundary-parallel. Hence, $T$ compresses in $M$. Let $D$ be any compressing disk. Surgering $T$ along $D$ yields a 2 -sphere $S$. By irreducibility of $M, S$ must bound a 3 -ball to one side. If the 3 -ball does not contain $D$, then $T$ bounds a solid torus in $M$. However, since $T$ must bound a knot exterior in $M^{\prime}$, there would be no boundary components of $M$ to either side of $T$, a contradiction. Hence the 3 -ball contains $D$. In fact, the 3 -ball is obtained by gluing a 2 -handle that is a neighborhood of $D$ to the knot exterior $Q$.

We will try to eliminate all intersection curves between $T$ and $P$. Trivial intersection curves on $T$ and $P$ are easily removed. We will assume that we have isotoped $T$ and $P$ to minimize the number of intersection curves.

Suppose first that there is an intersection curve concentric with $\phi_{1}$ or $\phi_{2}$ on $P$. Then there exists an annulus $A$ on $P$ with one boundary component a nontrivial curve on $T$ and the other boundary component a nontrivial curve on $\partial M$. Surgering $T$ along $A$ yields an annulus properly embedded in $M$ that must be boundary-parallel. Hence $T$ must be boundary-parallel in $M$. However, $g$ then lies in an $S^{1} \times S^{1} \times I$, one boundary component of which is $T$ and the other boundary component of which is in $\partial M$, contradicting condition (a). Hence, neither $\phi_{1}$ nor $\phi_{2}$ can have concentric intersection 
curves on $P$.

Thus, all the intersection curves on $P$ are concentric with $\sigma$. Suppose that there are intersection curves. Choose two adjacent curves on $T$. On $P$, replace the annulus that they bound with the annulus on $T$ that they bound. The resultant pair of pants intersects $T$ in fewer curves and still contains $\alpha$. Repeating this process results in a pair of pants $P^{\prime}$ that has the same boundary as $P$, does not intersect $T$ and contains $\alpha$.

The pair of pants $P$ must intersect the compressing disk $D$ since $g$ must intersect $D$ in order that $T$ be incompressible in $M^{\prime}$. We can remove all simple closed curves in $P \cap D$. Suppose there is an arc in the intersection. The arc must run to and from $\sigma$ on $P$. Suppose that the arc can be isotoped into $\sigma$ in $P$. Then we can isotope $P$ to intersect $D$ fewer times. Repeating this process, we can eliminate all intersection arcs on $P$ which are isotopic into $\sigma$. Since $P$ must intersect $D$, there must be at least one intersection arc on $P$ which is not isotopic into $\sigma$. This intersection arc will intersect $\alpha$. Hence $\alpha$ intersects $D$. Therefore, $T$ is incompressible in $M-\stackrel{\circ}{N}(\alpha)$, contradicting our hypothesis.

Thus, conditions (a), (b), (c) and (d) of the hypotheses of Theorem 2.1 are satisfied when we take the curve $g$ to be the knot $K$ in $M$. Therefore, $M-\stackrel{\circ}{N}(g)$ does have a hyperbolic structure, as we wished to show.

It is not difficult to come up with examples where the curve that we remove in order to introduce an appropriate pair of pants must be modified in order to satisfy conditions (a) and (c) as described in the proof of the above theorem.

Let $M$ be a compact 3-manifold such that $\stackrel{\circ}{M}$ is a noncompact finite volume hyperbolic 3-manifold. Let $C$ be a set of disjoint cusps in $\stackrel{\circ}{M}$. Then $\stackrel{\circ}{M}-\stackrel{\circ}{C}$ is homeomorphic to $M$. Therefore, if $p$ is the projection map from $H^{3}$ to $\stackrel{\circ}{M}$, then we can also think of $p$ as a map from $H^{3}-p^{-1}(\stackrel{\circ}{\boldsymbol{C}})$ onto $M$.

LEMMA 2.3. Let $M$ be a compact 3-manifold such that $\stackrel{\circ}{M}$ is a noncompact finite volume hyperbolic 3-manifold. Choose a set of cusps for $\stackrel{\circ}{M}$ which do not intersect. Lift to hyperbolic 3-space. 
Choose a particular pair of horoballs in the cover of the cusps. Take the arc $\alpha^{\prime}$ which is the shortest path between them. If it projects to a simple arc $\alpha$ in $M$, then $\alpha$ is essential in $M$ and $M-\stackrel{\circ}{N}(\alpha)$ does not contain a knot exterior with incompressible boundary.

Proof. If $\alpha$ were homotopic to an arc $\beta$ in $\partial M$, then both $\alpha$ and $\beta$ could be lifted to a pair of arcs in $H^{3}$ which begin and end at the same pair of points. However, $\beta$ will lift to an arc which stays in the same horosphere, while $\alpha^{\prime}$ was chosen to begin and end on distinct horospheres.

Suppose that $M-\stackrel{\circ}{N}(\alpha)$ contained a knot exterior $R$ with incompressible boundary. We can isotope $R$ to avoid the cusps in $\stackrel{\circ}{M}$. Since $\stackrel{\circ}{M}$ is hyperbolic, the boundary torus $T$ of the knot exterior must either be boundary-parallel or compressible in $M$. If $T$ were boundary-parallel, then $\alpha$ would be homotopic into $M$, contradicting the above paragraph.

We can therefore assume that $T$ compresses along a disk $D$ in $M-\stackrel{\circ}{R}$. We can isotope $D$ to miss the cusps. Since it is bounded by a sphere and it is contained in a cusped hyperbolic 3 -manifold, the manifold $R \cup N(D)$ must be a 3 -ball $B$ in $M$. The 3 -ball $B$ lifts to a disjoint set of 3-balls in $H^{3}$. Let $B^{\prime}$ be a particular one of these 3-balls in $H^{3}$. Then $B^{\prime}$ must contain a homeomorphic copy of $R$, call it $R^{\prime}$. The boundary of $R^{\prime}$ must be incompressible in $H^{3}-\left(p^{-1}(\stackrel{\circ}{\boldsymbol{C}}) \cup p^{-1}(\stackrel{\circ}{N}(\alpha))\right)$. Note that $B$ is a finite volume submanifold of $M$ and therefore $B^{\prime}$ is also finite volume. Hence, we can choose a 3 -ball $E$ in $H^{3}-p^{-1}(\stackrel{\circ}{\boldsymbol{C}})$ that contains $B^{\prime}$ and such that any arc in $p^{-1}(\alpha)$ that is intersected by $E$ is isotopic into $\partial E$ in $E$. Then the fundamental group of $E-p^{-1}(\stackrel{\circ}{N}(\alpha))$ is a free group on a finite number of generators. $R^{\prime}$ is a knot exterior contained within it with incompressible boundary. Hence the fundamental group of $R^{\prime}$ must be a subgroup of a free group and hence be a free group itself by the Nielsen-Schreier Subgroup Theorem. However nontrivial knot exterior groups are never free, a contradiction.

THEOREM 2.4. Let $M$ be compact orientable 3-manifold with interior a noncompact finite volume hyperbolic 3-manifold. Choose 
any two nontrivial disjoint simple closed curves $\phi_{1}$ and $\phi_{2}$ on its boundary. Then $M$ has a parent manifold $M^{\prime}$ such that $\stackrel{\circ}{M}$ is hyperbolic and $\stackrel{\circ}{M}^{\prime}$ contains a totally geodesic thrice-punctured sphere, two punctures of which correspond to $\phi_{1}$ and $\phi_{2}$.

Proof. Let $C_{1}$ and $C_{2}$ be the two cusps corresponding to the torus boundary components in which $\phi_{1}$ and $\phi_{2}$ lie. It may be the case that $C_{1}$ and $C_{2}$ are the same cusp. Lift them to the corresponding set of disjoint horospheres in $H^{3}$. Choose a geodesic arc that has the shortest length among all geodesic arcs that pass from the boundary of a horoball covering $C_{1}$ to the boundary of a horoball covering $C_{2}$. This arc must project to a simple arc in $M$. We can apply Theorem 2.2 and Lemma 2.3, to obtain a simple closed curve $g$ such that the interior of $M-\stackrel{\circ}{N}(g)$ is hyperbolic and $M-\stackrel{\circ}{N}(g)$ contains an essential pair of pants with two of its boundary components corresponding to $\phi_{2}$ and $\phi_{2}$. The interior of $M-\stackrel{\circ}{N}(g)$ then contains an essential thrice-punctured sphere, which, by the results of [1], must be totally geodesic.

From the algebraic point of view, we have:

COROLLARY 2.5. Every torsion free finite volume noncompact Kleinian group can be obtained by adding one relator to a nonelementary Kleinian group that contains a Fuchsian subgroup.

3. Proofs. We now reprove Myer's result from [16] utilizing our approach.

THEOREM 3.1. Every closed orientable 3-manifold contains a knot with hyperbolic complement.

Proof. In the proof of Theorem B of $[\mathbf{2 0}]$ (or see $[\mathbf{1 1}]$ and remove a neighborhood of the branching set), Thurston proved that every closed 3-manifold can be obtained by surgery on a finite volume hyperbolic 3-manifold. In fact, he proved that every closed 3-manifold can be obtained by surgery on a hyperbolic link in $S^{3}$. (An alternative proof of this fact appears in [2, p. 126].) 
Therefore, if $M_{0}$ is a given closed 3-manifold, we can choose a 3-manifold $M_{1}$ with hyperbolic interior from which $M_{0}$ can be obtained by Dehn filling. Suppose that $M_{1}$ has $n$ boundary components, where $n>1$. We will prove that $M_{0}$ can be obtained by Dehn filling a 3-manifold with only $n-1$ boundary components and hyperbolic interior.

Choose any two boundary components $T_{1}$ and $T_{2}$ of $M_{1}$. Lemma 2.3 allows us to choose an essential arc $\alpha$ in $M_{1}$ running from $T_{1}$ to $T_{2}$ that satisfies the hypotheses of Theorem 2.2. Note that by choosing $\alpha$ as the shortest arc running between the two cusps, we are assured that it is a simple arc.

Choose $\phi_{1}$ and $\phi_{2}$ parallel to the surgery curves on $T_{1}$ and $T_{2}$ respectively. Form the curve $g$ as in the proof of Theorem 2.2 from $\alpha, \phi_{1}$ and $\phi_{2}$. Remove the curve $g$ from $M_{1}$ to obtain a hyperbolic parental manifold $M_{2}$ of $M_{1}$ containing a pair of pants $P$ with two boundary components $\phi_{1}$ and $\phi_{2}$.

In the hyperbolic structure on $\stackrel{\circ}{M}_{2}, \stackrel{\circ}{P}$ can be isotoped to a totally geodesic thrice-punctured sphere (cf. [1]). Cut the manifold $M_{2}$ open along $P$, turn one copy of $P$ a half-twist, switching the $\phi_{1}$ and $\phi_{2}$ boundary components, and reglue, noting that the resulting manifold $M_{3}$ has one fewer cusp. By Theorem 4.1 of $[1], \stackrel{\circ}{M}_{3}$ is hyperbolic with the same volume as $\stackrel{\circ}{M}_{2}$.

Suppose that the boundary component of $P$ that lies in $\partial N(g)$ is a $(u, v)$-curve on $N(g)$. Define $M_{3(a, b)}$ to be the manifold obtained by doing an $(a, b)$-surgery on the boundary component of $M_{3}$ corresponding to $g$. We will choose $(a, b)$ so that the $(a, b)$-curve crosses the $(u, v)$-curve exactly once. We will show that $M_{0}$ can then be obtained by Dehn filling the manifold $M_{3(a, b)}$.

We can think of $N(g)$ as a solid torus in $M_{0}$. Since two of the boundary components of $P$ are parallel to surgery curves that yield $M_{0}, P$ can be capped off in $M_{0}$ with a pair of disks $D_{1}$ and $D_{2}$ to yield a disk $D$ in $M_{0}$ with boundary in $\partial N(g)$. Let $B$ be the 3-ball in $M_{0}$ given by $N(D) \cup N(g)$. We will alter the surgery instructions that yield $M_{0}$ only within the 3 -ball $B$. Starting with $M_{1}$, we will do the prescribed Dehn fillings on all the boundary components except for $T_{1}$ and $T_{2}$. Each of $T_{1}$ and $T_{2}$ bound solid tori in $M_{0}$ which each cut through $B$ once as unknotted 1-handles. Glue $N\left(D_{1}\right)$ and 
$N\left(D_{2}\right)$ to the boundary of this 3-manifold, where these two regular neighborhoods are not as wide as $N(D)$. Call the resulting manifold $Q$. If we glued solid balls to the two spherical boundaries of $Q$, the result would be $M_{0}$. Instead, take two disjoint arcs properly embedded in the manifold, such that they are contained in $B$ and properly embedded in $Q$ and such that they criss cross in $B$ as in Figure $1 \mathrm{~b}$ ). Define $Q^{\prime}$ to be the manifold obtained by removing a regular neighborhood of the union of these two arcs from $Q$.

Then Dehn filling this single torus boundary in $Q^{\prime}$ with a surgery curve that is meridianal on one of the two arcs will yield $M_{0}$. The same holds true if we twist the two arcs as in Figure 1c).

However, the resulting manifold $Q^{\prime}$ is homeomorphic to $M_{3(a, b)}$, where $(a, b)$ is determined by the number of additional full twists in the two arcs and the fact this curve crosses the $(u, v)$-curve once. Note that we can find arbitrarily large values of $a$ and $b$ that are appropriate, as if an $\left(a_{0}, b_{0}\right)$-curve intersects the $(u, v)$-curve once, so does an $\left(a_{0}+n u, b_{0}+n v\right)$-curve for any integer $n$. For high

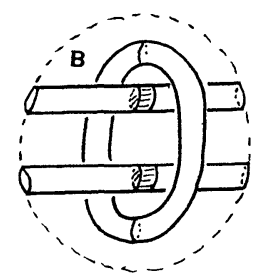

a)

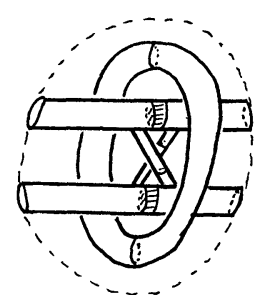

b)

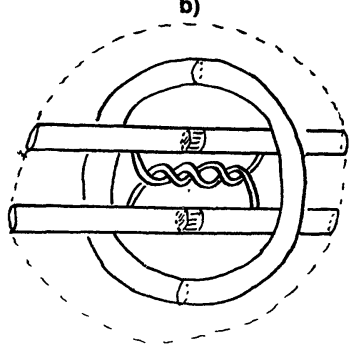

c)

FiguRE 1 
enough values of $(a, b), M_{3(a, b)}$ will be hyperbolic by Chapter 5 of [19]. Hence, this demonstrates that if $M_{0}$ comes from Dehn filling a finite volume hyperbolic 3 -manifold with $n$ cusps, where $n \geq 2$, we can in fact find a hyperbolic manifold with $n-1$ cusps from which it comes by Dehn filling. Iterating the process, we find a hyperbolic manifold with one cusp from which $M_{0}$ comes by a single Dehn filling. This is equivalent to showing that there exists a knot in $M_{0}$ with hyperbolic complement, the knot corresponding to the core curve of the solid torus that is glued on in the process of performing the Dehn filling.

In order to prove Theorem $1.1(\mathrm{a})$, we will need the next lemma.

Lemma 3.2. A link $L_{m}$ in $S^{3}$ with a projection as in Figure 2 has a hyperbolic complement with volume $2(m-1)(3.6638 \ldots)$ for $m \geq 2$, where $m$ is the number of components bounding twice-punctured disks.

Proof. Such a link complement can be obtained by cutting open $2(m-1)$ Whitehead link complements along embedded twice-punctured disks and gluing the results together in the appropriate way along the twice-punctured disks as in Figure 3. Repeating the process depicted there, and then cutting open along a single twicepunctured disk and twisting a half-twist before regluing yields the link depicted in Figure 2.

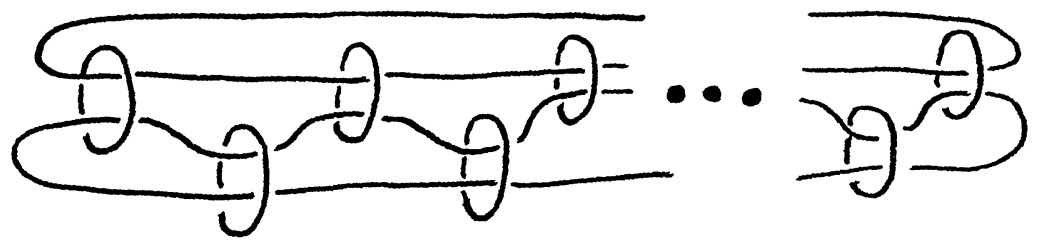

FIGURE 2 

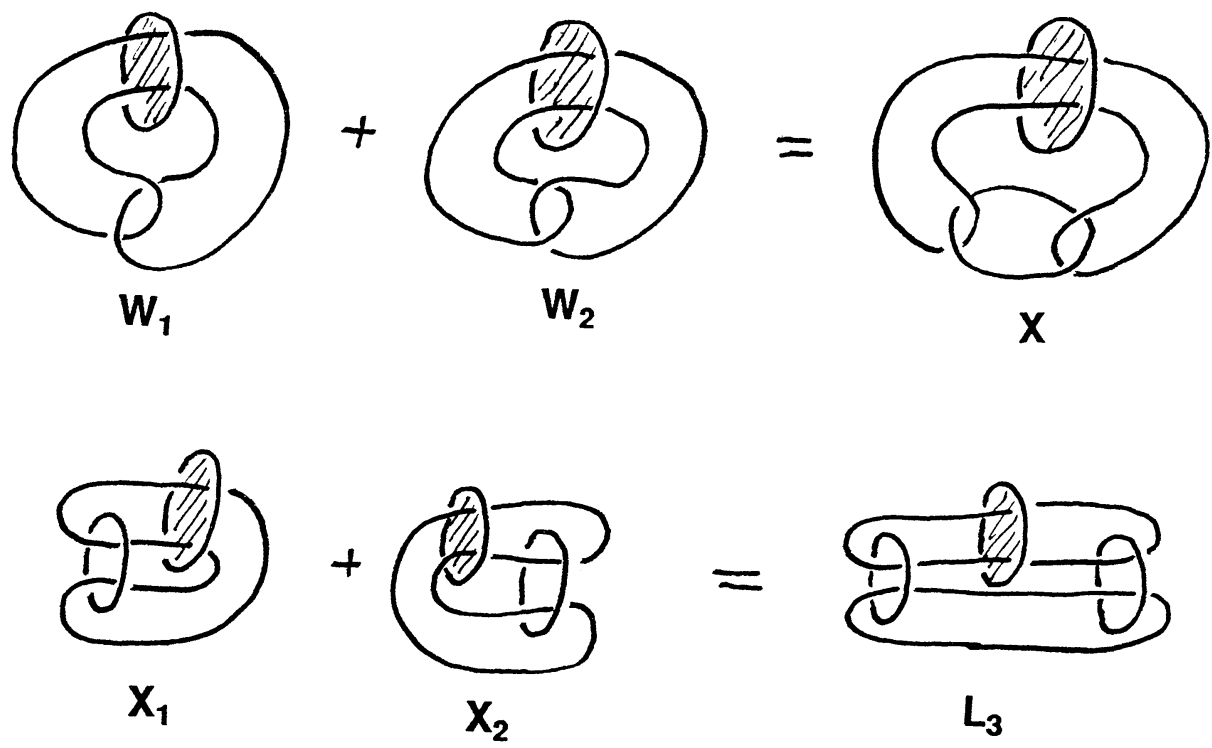

FiguRE 3

Corollaries 5.1 and 5.2 of [1] imply that the result is hyperbolic with volume $2(m-1)$ times the volume of the Whitehead link, where the volume of the Whitehead link is given approximately by 3.6638 .

We will now prove Theorem 1.1.

THEOREM 1.1 (a). Let $V_{0}$ be any positive real number. Then the set of 1-cusped hyperbolic 3-manifolds of volume greater than $V_{0}$ is a complete family of parent manifolds.

Proof of 1.1 (a). Given a closed orientable 3-manifold $M_{0}$, Theorem 3.1 allows us to find a 3 -manifold $M_{1}$ from which $M_{0}$ comes by a single Dehn filling, such that the interior of $M_{1}$ is a 1-cusped hyperbolic 3-manifold. We can realize $M_{1}$ as a submanifold of $M_{0}$ given by $M_{0}-\stackrel{\circ}{N}(K)$, where $K$ is a knot in $M_{0}$. As in Lemma 2.3, we can find a simple essential arc $\alpha$ in $M_{1}$. Letting $\phi_{1}$ and $\phi_{2}$ be parallel to the surgery curve on $M_{1}$, we can apply Theorem 2.4 to obtain a manifold $M_{2}$ from which $M_{0}$ comes by two Dehn fillings. The interior of $M_{2}$ is a 2-cusped hyperbolic 3-manifold containing a thrice-punctured sphere.

Let $P$ be the pair of pants in $M_{2}$ bounded by $\phi_{1}, \phi_{2}$ and a curve in $\partial N(g)$. If we perform the Dehn filling on the boundary component of $M_{1}, P$ can be capped off along the two boundary components $\phi_{1}$ 
and $\phi_{2}$ to obtain a disk $D$ in $M_{0}$. Let $B$ be a regular neighborhood of $D \cup N(g)$ in $M_{0}$. Then $B$ is a ball in $M_{0}$.

We note that we can modify the surgery instructions for $M_{0}$ within this ball as follows. Currently, $K$ intersects $B$ in two properly embedded unknotted arcs. We could replace $K$ within $B$ by a rational tangle. If $K^{\prime}$ were the resultant new knot within $M_{0}$, then $M_{0}$ would come from Dehn filling $M_{0}-\stackrel{\circ}{N}\left(K^{\prime}\right)$ such that the surgery curve were still a meridian on $K^{\prime}$.

By Theorem 4.5 of [1] and Lemma 3.2, we can cut $S^{3}-L_{m}$ open along one of the twice-punctured disks, cut $M_{2}$ open along $P$ and glue the two manifolds together along these surfaces. The resulting manifold $M_{3}$ will have volume equal to

$$
\operatorname{vol}\left(M_{2}\right)+2(m-1)(3.6638 \ldots) \text {. }
$$

For each of the trivial loops in $L_{m}$, we can do a $(1, p)$ surgery for $p$ very high in order to ensure that the resulting manifold $M_{4}$ is hyperbolic and has volume arbitrarily close to $M_{3}$ (cf. Chapters 5 and 6 of [19]). By choosing $m$ large enough, we can be assured that $M_{4}$ has volume as large as we would like. However, $M_{4}$ is given by $M_{0}-N\left(K^{\prime}\right)$ where $K^{\prime}$ is identical to $K$ outside of $B$ and has a rational tangle inside $B$. Hence $M_{0}$ can be obtained by Dehn filling $M_{4}$, where the interior of $M_{4}$ is a hyperbolic 3-manifold of one cusp with arbitrarily high volume.

Note that the above argument did not rely on the fact that $M_{0}$ was a closed manifold. Hence we have the following corollary.

CoROllary 3.3. Given a finite volume hyperbolic 3-manifold $M$, there is no bound on $\left|\operatorname{vol}\left(M^{\prime}\right)-\operatorname{vol}(M)\right|$, where $M^{\prime}$ is a hyperbolic parent of $M$.

THEOREM $1.1(\mathrm{~b})$. Let $V_{1}$ be any positive real number. Then the set of 1-cusped hyperbolic 3-manifolds of cusp volume greater than $V_{1}$ is a complete family of parent manifolds.

Proof of 1.1 (b). Let $M_{0}$ be an arbitrary closed hyperbolic 3-manifold. We repeat the construction in the proof of Theorem 1.1 (a), and see what we can say about the cusp volume of the resulting manifold. 
Choose the cusp $C_{1}$ corresponding to $K$ in $M_{2}$ to be a maximal cusp. Let $C_{2}$ be the remaining cusp in $M_{2}$. Lifting to hyperbolic space, choose a geodesic plane $\widetilde{P}$ that covers the thrice-punctured sphere $P$ in $M_{2}$. If there exist horoballs covering $C_{1}$ or $C_{2}$ that are not centered on the boundary of $\widetilde{P}$ but that intersect $\widetilde{P}$, shrink all of the horoballs covering either cusp equivariantly until no such intersections occur. Let $C_{1}$ and $C_{2}$ now denote the resulting cusps in $M_{2}$.

Let $P^{\prime}$ be a particular choice of one of the totally geodesic twicepunctured disks in $S^{3}-L_{m}$. Let $C_{2}^{\prime}$ be the cusp that corresponds to its boundary and let $C_{1}^{\prime}$ be the cusp that punctures it twice. Let $\widetilde{P}^{\prime}$ be a geodesic plane in $H^{3}$ that covers $P^{\prime}$. Since $S^{3}-L_{m}$ is constructed from a set of copies of the Whitehead link complement, the only horoballs covering cusps of $S^{3}-L_{m}$ that intersect $\widetilde{P}^{\prime}$ are those horoballs centered on the boundary of $\widetilde{P}^{\prime}$.

Cutting $M_{2}$ open along $P$, cutting $S^{3}-L_{m}$ open along $P^{\prime}$ and then gluing each copy of $P$ to a copy of $P^{\prime}$ so that the cusps $C_{1}$ and $C_{1}^{\prime}$ are matched, and the cusps $C_{2}$ and $C_{2}^{\prime}$ are matched, we obtain the manifold $M_{3}$. Let $C_{1}^{\prime \prime}$ and $C_{2}^{\prime \prime}$ represent the corresponding cusps in $M_{3}$.

We would like to obtain a valid horoball covering for $C_{1}^{\prime \prime}$. Since we have already eliminated the horoballs that poke through the corresponding geodesic plane, we need only fit together the horoballs centered on the boundary of the geodesic planes. However, any two horoballs covering the cusp $C_{1}$ in $M_{2}$ with centers on the boundary of $\widetilde{P}$ are identified by the subgroup of isometries fixing $\widetilde{P}$. Similarly, a pair of horoballs covering $C_{1}^{\prime}$ in $S^{3}-L_{m}$ with centers on the boundary of $\widetilde{P}^{\prime}$ are identified by the subgroup of isometries fixing $\widetilde{P}^{\prime}$. When we glue the two manifolds together along the twice-punctured disks, we identify the subgroup of isometries fixing $\widetilde{P}$ with the subgroup of isometries fixing $\widetilde{P}^{\prime}$. Hence, it is enough to make sure that one horoball covering $C_{1}$ with center on the boundary of $\widetilde{P}$ is the same size as the horoball that it is identified to that covers $C_{1}^{\prime}$ and that has center on the boundary of $\widetilde{P}^{\prime}$. By either shrinking the horoballs covering $C_{1}$ in $M_{2}$ or shrinking the horoballs in $S^{3}-L_{m}$ covering $C_{1}^{\prime}$, we can be assured that the horoballs will fit together. We obtain a cusp of some finite volume in $M_{3}$. It is not necessarily a maximal cusp, but its volume does give a lower bound to the 
maximal cusp volume of this cusp in $M_{3}$.

Once the cusp in $S^{3}-L_{m}$ has been shrunk to the appropriate size for gluing to the cusp in $M_{2}$, no additional shrinkage of the cusp is necessary as $m$ increases. Each additional copy of a Whitehead link complement that has been cut open along a twice-punctured disk and that we glue in contributes the same fixed amount to this particular cusp. Hence, the volume in this cusp increases linearly with $m$. In particular, as $m$ approaches $\infty$, the volume in the maximal cusp approaches $\infty$.

Now, we repeat the end of the construction in the proof of Theorem 1.1 (a). Performing high enough $(1, p)$-Dehn fillings on all the cusps except for $C_{1}^{\prime \prime}$ in $M_{3}$ yields a hyperbolic manifold $M_{4}$ such that the maximal cusp volume of its one cusp is arbitrarily close to the maximal cusp volume of $C_{1}^{\prime \prime}$ in $M_{3}$. This follows from the fact that the hyperbolic structures on a sequence of manifolds obtained by Dehn filling cusps of a hyperbolic 3-manifold geometrically converge to the hyperbolic structure on the surgered manifold. (See [19] or [6].) Thus, $M_{4}$ is a one-cusped hyperbolic 3-manifold of arbitrarily high cusp volume from which $M_{0}$ can be obtained by a single Dehn filling.

We have the following topological corollary.

COROLlaRY 3.4. There exists a complete family of parent manifolds consisting of one-cusped hyperbolic 3-manifolds such that at most one Dehn filling on each manifold yields a manifold with finite fundamental group.

Proof. The Thurston-Gromov " $2 \pi$ " Theorem states that a Dehn filling of a cusped hyperbolic 3-manifold along a curve of length at least $2 \pi$ in a cusp boundary of a finite volume hyperbolic 3-manifold yields a closed manifold of negative curvature.(See [5] and [8].) Such a manifold cannot have finite fundamental group. However, the volume $V$ in a cusp is equal to $\left(d_{1} d_{2} \sin \phi\right) / 2$, where $d_{1}$ is the length of the shortest nontrivial curve in the cusp boundary, $d_{2}$ is the length of the shortest nontrivial curve that is not a multiple of the shortest curve, and $\phi$ is the angle between the translation directions of the two parabolic isometries that are generated by these two curves. 
Assuming that there exists a curve in the cusp boundary of a one-cusped hyperbolic 3-manifold for which the corresponding Dehn filling yields finite fundamental group, it must be that $1 \leq d_{1} \leq 2 \pi$. Thus, $d_{2} \geq V / \pi$. If $V$ is chosen to be greater than $2 \pi^{2}, d_{2}$ will be greater than $2 \pi$. Thus, it suffices to take all one-cusped orientable hyperbolic 3-manifolds with cusp volumes of at least 20 in order to obtain a complete family of 3 -manifolds such that at most one Dehn filling on each yields a finite fundamental group.

In particular, if $M$ is a counterexample to the Poincare conjecture, it comes from Dehn filling a hyperbolic 3-manifold with one cusp, such that every other Dehn filling of that cusp yields infinite fundamental group.

Note that one way of stating the conjectured Property $P$ for knots in $S^{3}$ is that at most one Dehn filling on any given knot exterior in $S^{3}$ yields a trivial fundamental group. Corollary 3.5 gives us a complete family of parent manifolds that satisfy this statement of Property $P$.

We also note that in proving Theorem 1.1 (b), we proved the additional fact that any closed 3 -manifold $M$ contains a knot such that the knot is hyperbolic and such that the Dehn filling that corresponds to filling the knot back in to obtain $M$ is a Dehn filling along the shortest nontrivial curve in the cusp boundary. This follows from the fact that as we increase $m$ by adding in additional copies of the Whitehead link complement, we extend the lengths of all of the simple closed curves in the cusp that are linearly independent from the Dehn filling curve without affecting the length of the Dehn filling curve. This is of interest, in light of the fact that Craig Hodgson and Jeff Weeks (c.f.[12] ) have discovered an example of a hyperbolic knot in $S^{3}$ such that the meridian curve is not the shortest curve in a cusp boundary.

THEOREM 1.1 (c). The set of 2-cusped hyperbolic 3-manifolds containing embedded totally geodesic surfaces is a complete family of ancestor (actually grandparent) manifolds.

Proof of 1.1 (c). This result follows immediately from Theorems 2.4 and 3.1 . 
The following algebraic corollary is immediate.

COROLlaRY 3.5. Any fundamental group of a closed oriented 3-manifold can be obtained by adding at most 2 relators to a nonelementary Kleinian group that contains a Fuchsian subgroup.

THEOREM 1.1 (d). For any positive integer $N$, the set of hyperbolic 3-manifolds, each of which shares its volume with $N$ or more other hyperbolic 3-manifolds, is a complete family of ancestor manifolds.

Proof of 1.1 (d). Given $N$, let $M_{0}$ be a closed orientable 3-manifold. Then $M_{0}$ can be obtained by Dehn filling a 3-manifold $M_{1}$ such that its interior is a 1-cusped hyperbolic 3-manifold. Theorem 2.2 allows us to find a knot $g$ in $M_{1}$ such that $M_{1}-\stackrel{\circ}{N}(g)$ has hyperbolic interior and such that there is an essential pair of pants $P$ in $M_{1}-\stackrel{\circ}{N}(g)$ so that $\stackrel{\circ}{P}$ is totally geodesic in the hyperbolic interior and so that two boundary components of $P$ are parallel to the surgery curve on $\partial M_{1}$ that yields $M_{0}$. Let $M_{2}=M_{1}-\stackrel{\circ}{N}(g)$.

Let $L_{n}$ be the link appearing in Figure 4. Then $L_{n}$ is an augmented alternating link and hence, $S^{3}-L_{n}$ is hyperbolic by [2]. Cut $M_{2}$ open along $P$, yielding two copies of $P$, denoted $P_{1}$ and $P_{2}$. Cut $S^{3}-L_{n}$ open along the twice-punctured disk $D$, yielding two copies of $D$, denoted $D_{1}$ and $D_{2}$. Let $M_{3}$ be a manifold obtained by gluing these two manifolds together along $P_{1}$ and $D_{1}$ and also along $P_{2}$ and $D_{2}$. The original manifold $M_{0}$ can be obtained from Dehn filling the resulting manifold $M_{3}$.

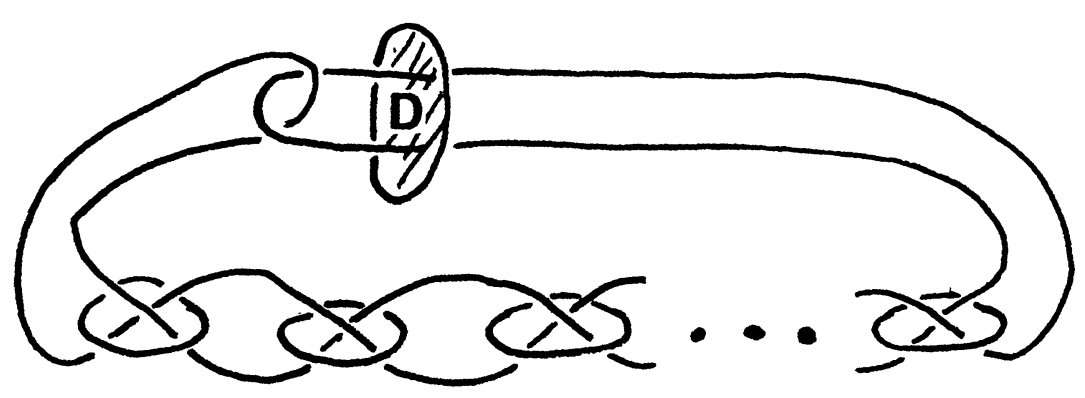

Figure 4 
However, by cutting open along twice-punctured disks in $M_{3}$ corresponding to the twice-punctured disks in $S^{3}-L_{n}$, twisting a halftwist and then regluing, we can obtain numerous manifolds, all of which are hyperbolic and all of which have the same volume by Theorem 4.1 of [1]. However, they cannot be homeomorphic as they have different numbers of cusps. By choosing $n$ large enough we are assured of any desired number of manifolds, all having the same volume as $M_{3}$.

\section{Bibliography}

[1] C. Adams, Thrice-Punctured Spheres in Hyperbolic 3-Manifolds, Trans. of A.M.S., 287:2 (1985), 645-656.

[2] C. Adams, Augmented Alternating Link Complements are Hyperbolic, in Low-dimensional Topology and Kleinian Groups, ed. by D. Epstein, London Math.Soc. Lecture Note Series 112, Cambridge University Press (1986), 115-130.

[3] J. W. Alexander, A lemma on systems of knotted curves, Proc. Natl. Acad. Sci. USA, 9 (1923), 93-95.

[4] R. H. Bing, Necessary and sufficient conditions that a 3-manifold be $S^{3}$, Ann. of Math., 68 (1958), 17-37.

[5] S. Bleiler and C. Hodgson, Spherical space forms and Dehn filling, preprint.

[6] R. C. Canary, D.B.A. Epstein and P. Green, Notes on Notes of Thurston, Analytical and Geometrical aspects of hyperbolic space, (Coventry/Durham 1984) 3-92, London Math. Soc. Lecture Notes, 111, Cambridge Univ. press, 1987.

[7] F. Gonzalez-Acuna, unpublished.

[8] M. Gromov and W. Thurston, Pinching constants for hyperbolic manifolds, Invent. Math., 89 (1987), 1-12.

[9] W. Haken, Theorie der Normäl Flachen, Acta Math., 105 (1961), 245375 .

[10] J. Hempel, 3-Manifolds, Annals of Mathematics Studies 86, Princeton University Press, 1976.

[11] H. Hilden, M. T. Lozano, J. M. Montesinos, On knots that are universal, Topology, 24:4 (1985), 499-504.

[12] C. Hodgson and J. Weeks, private communication.

[13] W. Jaco, Lectures on three-manifold topology, Regional Conference Series in Mathematics 43, American Mathematical Society, 1980.

[14] W.B.R.Lickorish, A representation of orientable combinatorial three-manifolds, Annals of Math., 76 (1962), 531-540.

[15] R. Myers, Open book decompositıons of 3-manifolds, Proc. Am. Math. Soc., 72 (1978), 397-402. 
[16] R. Myers, Simple knots in compact, orientable 3-manifolds, Trans. of A.M.S., 273:1 (1982), 75-91.

[17] R. Myers, Excellent 1-manifolds in compact 3-manifolds, preprint.

[18] D. Rolfsen, Knots and Links, Publish or Perish, Inc., Berkeley, 1976.

[19] W. Thurston, The Geometry and Topology of 3-Manifolds, lecture notes, Princeton University, 1978.

[20] W. Thurston, Universal Links, preprint (1982).

[21] A.D. Wallace, Modifications and cobounding manifolds, Canad. J. Math., 6 (1960), 268-279.

Received April 8, 1992, revised February 4, 1993, and accepted for publication March 12, 1993. This work was supported in part by DMS-9000937.

Williams College

Williamstown, MA 01267

E-mail address: Colin.Adams@williams.edu 


\section{CONTENTS}

S. Abdulali, Conjugates of strongly equivariant maps .............. 207

C. Adams, Dehn filling hyperbolic 3-manifolds ...................... 217

S. Berman and B. Cox, Enveloping algebras and representations of toroidal Lie algebras.......................................... 239

B. Cox, Verma modules induced from nonstandard Borel subalgebras . 269

C. H. FitzGerald and C. R. Thomas, Some bounds on convex mappings in several complex variables..., ...................... 295

P. H. Loi, On the derived towers of certain inclusions of type $I I I_{\lambda}$ fac-

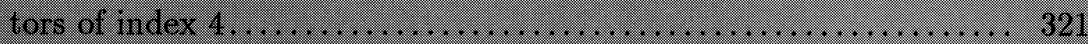

F. Morgan, Soap bubbles in $\mathbb{R}^{2}$ and in surfaces..................... 347

M. Shiffman, Partially measurable sets in measur spaces ............. 363 


\section{PACIFIC JOURNAL OF MATHEMATICS}

Volume $165 \quad$ No. $2 \quad$ October 1994

Conjugates of strongly equivariant maps

207

SALMAN ABDULALI

Dehn filling hyperbolic 3-manifolds

217

COLIN C. ADAMS

Enveloping algebras and representations of toroidal Lie algebras

239

STEPHEN BERMAN and BEN COX

Verma modules induced from nonstandard Borel subalgebras

269

BEN COX

Some bounds on convex mappings in several complex variables

295

CARL HANSON FitzGERALD and CAROLYN R. THOMAS

On the derived towers of certain inclusions of type $I I I_{\lambda}$ factors of

321 index 4

PHAN HUNG LOI

Soap bubbles in $\mathbb{R}^{2}$ and in surfaces

347

FRANK MORGAN

Partially measurable sets in measure spaces

MAX SHIFFMAN 